Europhys. Lett., 55 (4), pp. 580-586 (2001)

\title{
Gas-liquid phase coexistence in colloidal suspensions?
}

\author{
H. H. von GrünberG $\left.{ }^{1}{ }^{*}\right)$, R. van RoiJ ${ }^{2}$ and G. Klein ${ }^{1}$ \\ 1 Fakultät für Physik, Universität Konstanz - 78457 Konstanz, Germany \\ 2 Institute for Theoretical Physics, Utrecht University \\ Princetonpln 5, 3584 CC Utrecht, The Netherlands
}

(received 4 January 2001; accepted in final form 30 May 2001)

PACS. 82.70.Dd - Colloids.

PACS. $64.10 .+\mathrm{h}$ - General theory of equations of state and phase equilibria.

PACS. 64.60. Cn - Order-disorder transformations; statistical mechanics of model systems.

\begin{abstract}
We describe a charge-stabilized colloidal suspension within a Poisson-Boltzmann cell model and calculate the free energy as well as the compressibility as a function of colloidal density. The same quantities are also calculated from the linearized Poisson-Boltzmann equation. Comparing nonlinear with linear theory, we test the quality of different linearization schemes. For concentrated suspensions, linearization about the Donnan potential is shown to be preferable to standard Debye-Hückel linearization. We also show that the volume term theory proposed earlier follows from a linearization about the Donnan potential. Using this linearization scheme, we find a gas-liquid phase coexistence in linear, but not in nonlinear theory. This result may imply that predictions of a spinodal instability in highly de-ionized colloidal suspensions are spurious.
\end{abstract}

Suspensions of charged colloidal particles at low salt concentrations have attracted much attention recently, mainly because of experimental claims of attractive interactions between like-charged colloids and the possibility of a gas-liquid phase transition [1-3]. Such phenomena cannot be explained by the standard and well-accepted DLVO theory [4], which predicts that screened-Coulomb repulsions between the like-charged colloids completely mask the van der Waals attractions in the low-salt regime of interest here. On this basis it is often argued that there is no cohesive energy that can stabilize a liquid phase. It is important to realize, however, that such a consideration is based on the implicit assumption that the (effective) interaction Hamiltonian of the colloids is a sum of pair potentials, i.e. that three- and more-body interactions are being ignored. This assumption is expected to be valid at high salt concentrations, where the screening length $\lambda$ is much shorter than the colloidal diameter $a$, but may well fail if $\lambda \simeq a$, i.e. at salt concentrations in the micro-mole regime. Recent attempts to effectively take into account these higher-body interactions (in the form of density-dependent "volume terms" that include the free energy of double layers with their "own" colloidal particle) are based on i) considering the total free energy of the suspension, including the "volume terms" and ii) including the counterion density to the screening, i.e. the screening becomes colloiddensity-dependent [5-8]. The combination of these two ingredients can explain (some of) the experimental observations qualitatively, since the resulting free energy gives rise to phase coexistence of a dilute (gas) phase with dense (liquid or crystal) phases; the cohesive energy that stabilizes the dense phases is provided by the Coulomb energy of the relatively compressed

$\left(^{*}\right)$ E-mail: Hennig.vonGruenberg@uni-konstanz.de

(C) EDP Sciences 
double layers in the dense phases at the sufficiently low salt concentration [7]. The theories of refs. [5-8] are, however, linearized Poisson-Boltzmann theories, and hence highly approximate. Moreover, even as a linearized theory, they are not undisputed since the traditional DLVO theory (which is also linear) does not include the counterion contribution to the screening.

In this paper we show that the counterion contribution to the screening follows naturally from linearizing the Poisson-Boltzmann equation about the Donnan potential, while the traditional DLVO result is obtained with a linearization about zero potential. We also investigate the quality of several linearization schemes compared to the nonlinear Poisson-Boltzmann scheme. For computational reasons the latter is calculated numerically in the simplified geometry of a spherical Wigner-Seitz cell. It turns out that the linearization about the Donnan potential is excellent at high enough colloidal density, contrary to the traditional linearization scheme. At low colloid density, however, both linearization schemes seem to fail. Moreover, within the nonlinear theory we do not recover the gas-liquid coexistence that is predicted by the Donnan-linearized theory.

We consider a fluid of $N$ highly charged identical colloidal spheres suspended in a structureless medium of macroscopic volume $V$, dielectric constant $\varepsilon$, and temperature $T(\beta=1 / k T)$. The colloidal spheres, with coordinates $\boldsymbol{R}_{i}(i=1 \ldots N)$, have a radius $a$, a fixed charge $-Z e$, and hence a surface charge density $\sigma_{\mathrm{C}}=Z / 4 \pi a^{2}$. We assume that the suspension is in osmotic equilibrium with a reservoir of monovalent point charges $\pm e$ of total density $2 n_{s}^{r}$, chemical potential $\beta \mu_{s}=\log \left(n_{s}^{r} \Lambda^{3}\right)$, and Debye screening parameter $\kappa=\left[8 \pi \lambda_{\mathrm{B}} n_{s}^{r}\right]^{1 / 2}$, where $\Lambda$ is the thermal wavelength of the ions, and $\lambda_{\mathrm{B}}=e^{2} \beta / \varepsilon$ the Bjerrum length characterizing the medium. The thermodynamic potential $F\left(N, V, T ; \mu_{s}\right)$ of this system can formally be written as

$$
\exp [-\beta F]=\frac{1}{N ! \Lambda^{3 N}} \int \mathrm{d} \boldsymbol{R}^{N} \exp [-\beta \Omega(\{\boldsymbol{R}\})],
$$

where $\Omega(\{\boldsymbol{R}\})$ denotes the effective (many-body) Hamiltonian of the one-component system of quasi-particles ("dressed colloids") at positions $\{\boldsymbol{R}\}$. Within a mean-field approach $\Omega(\{\boldsymbol{R}\})$ can be written in terms of the equilibrium microion profiles $n_{ \pm}(\boldsymbol{r})=n_{s}^{r} \exp [\mp \phi(\boldsymbol{r})]$ and the (dimensionless) electrostatic potential $\phi(\boldsymbol{r})$ as

$$
\beta \Omega=\frac{1}{8 \pi \lambda_{\mathrm{B}}} \int_{G} \mathrm{~d} \boldsymbol{r}(\nabla \phi)^{2}+\sum_{\alpha= \pm} \int_{G} \mathrm{~d} \boldsymbol{r} n_{\alpha}\left(\log n_{\alpha} \Lambda^{3}-1\right)-\int_{G} \mathrm{~d} \boldsymbol{r}\left[\beta \mu_{s}\left(n_{+}+n_{-}\right)-2 n_{s}^{r}\right],
$$

where $G$ denotes that part of the volume $V$ exterior to the colloidal spheres. The first term in eq. (2) is the electrostatic energy, the second part the microion entropy, and the third term signifies the grand-canonical treatment of the microions. The evaluation of $\Omega$ thus requires $\phi(\boldsymbol{r})$. In principle $\phi(\boldsymbol{r})$ follows from the Poisson-Boltzmann (PB) equation

$$
\begin{aligned}
\nabla^{2} \phi(\boldsymbol{r}) & =\kappa^{2} \sinh \phi(\boldsymbol{r}), & & \boldsymbol{r} \in G, \\
\boldsymbol{n}_{i} \cdot \nabla \phi & =4 \pi \lambda_{\mathrm{B}} \sigma_{\mathrm{C}}, & & \boldsymbol{r} \in \partial G_{\boldsymbol{R}_{i}},
\end{aligned}
$$

where we assume constant-charge boundary conditions, and where $\partial G_{\boldsymbol{R}_{i}}$ represents the surface of the $i$-th colloid with outward pointing surface normal $\boldsymbol{n}_{i}$. In practice, however, the PB equation cannot be solved analytically, and even a numerical solution of eq. (3) for arbitrary $\{\boldsymbol{R}\}$ poses a formidable challenge. Moreover, even if $\phi(\boldsymbol{r})$ and hence $\Omega(\{\boldsymbol{R}\})$ were known, it would be next to impossible to calculate $F$ from eq. (1).

Here we circumvent these problems by selecting only one colloidal configuration for which we calculate $\Omega$. We choose the crystalline fcc configuration, $\{\boldsymbol{R}\}_{\text {fcc }}$, which we expect to be 
the most important one because the colloids have a repulsive effective interaction and thus try to have the maximum distance to each other. We hence approximate eq. (1) by

$$
\exp [-\beta F]=\exp \left[-\beta \Omega\left(\{\boldsymbol{R}\}_{\mathrm{fcc}}\right)\right] \frac{1}{N ! \Lambda^{3 N}} \int \mathrm{d} \boldsymbol{R}^{N} .
$$

This leads directly to

$$
F=N f^{\text {id }}+\Omega\left(\{\boldsymbol{R}\}_{\text {fcc }}\right)=N\left(f^{\text {id }}+f^{\text {ex }}\right),
$$

with the ideal-gas free energy per particle $\beta f^{\text {id }}=\log n_{p} \Lambda^{3}-1$, where $n_{p}=N / V$ the colloid density, and with $f^{\text {ex }}=\Omega\left(\{\boldsymbol{R}\}_{\text {fcc }}\right) / N$ the excess (over ideal) free energy per colloidal particle. Due to the symmetry of the fcc configuration, $\Omega\left(\{\boldsymbol{R}\}_{\text {fcc }}\right) / N$, and hence $f^{\text {ex }}$, is given by eq. (2), but with i) the integration volume $G$ replaced by $G_{\text {cell }}$, the Wigner-Seitz cell of the fcc lattice, and ii) $\phi(\boldsymbol{r})$ the solution of the $\mathrm{PB}$ cell equation:

$$
\begin{aligned}
\nabla^{2} \phi(\boldsymbol{r}) & =\kappa^{2} \sinh \phi(\boldsymbol{r}), & & \boldsymbol{r} \in G_{\text {cell }}, \\
\boldsymbol{n}_{1} \cdot \nabla \phi & =4 \pi \lambda_{\mathrm{B}} \sigma_{\mathrm{C}}, & & \boldsymbol{r} \in \partial G_{\boldsymbol{R}_{1},}, \\
\boldsymbol{n}_{1} \cdot \nabla \phi & =0, & & \boldsymbol{r} \in \partial G_{\text {cell }},
\end{aligned}
$$

which is formulated here for the colloid at $\boldsymbol{R}_{1}$. Dividing the volume into cells to describe the fluid state might seem an inappropriate procedure at first glance, but this idea has had a long tradition in liquid-state physics [9] and is also well known in the theory of effective colloidal interactions [10]. To appreciate the cell approach, it is essential to realise that every macroion of the suspension is surrounded by a closed surface with a vanishing normal component $E_{n}$ of the electric field, regardless the specific colloidal configuration $\{\boldsymbol{R}\}$ under consideration. The surface integral of $E_{n}$ over such an enclosing surface is zero, and the cell defined by this surface is thus electrically neutral. This shows that the colloidal suspension can be very naturally divided into space-filling electro-neutral cells, not only in the crystalline phase, but also in the fluid phase, regardless of the colloid density. In the fluid phase, these cells differ from each other by volume and shape, whereas they are all identical in the crystalline phase. The average cell volume $\Delta$, however, is in any case given by the colloid density through $\Delta=1 / n_{p}$. The specific form of the cellular structure of the suspension is determined by the colloidal configuration $\{\boldsymbol{R}\}$. The central approximation in the cell theory is therefore not the assumption of the existence of cells as such, but the assumption that the cells in the fluid phase have all equal volume and shape. The extra boundary condition on the cell edge $\partial G_{\text {cell }}$ in eq. (6) then effectively accounts for the presence of the other colloids in the system. A further simplifying approximation is to consider the shape of this cell as a sphere of radius $R$ with $4 \pi R^{3} n_{p} / 3=1$. With all cells being identical, this approximation has the major advantage that we need to calculate $\phi$ in one cell only. The resulting PB cell equation for $\phi(r)$ is radially symmetric, and thus easily solvable numerically on a one-dimensional grid. We thus obtain

$$
\beta f^{\mathrm{ex}}=\frac{-Z}{2}\left(\phi(a)-\frac{Z \lambda_{\mathrm{B}}}{a}\right)+n_{s}^{r} 4 \pi \int_{a}^{R} r^{2}[\phi \sinh \phi-2 \cosh \phi+2] \mathrm{d} r,
$$

which follows from eq. (2) using Green's identity, the boundary conditions of eq. (6), $n_{ \pm}=$ $n_{s}^{r} e^{\mp \phi}$, and $\beta \mu_{s}=\log \left(n_{s}^{r} \Lambda^{3}\right)$. For later convenience, we subtracted the Coulomb self-energy of the colloidal charges $Z^{2} \lambda_{\mathrm{B}} / 2 a$. We can thus compute the free energy of the dressed colloids as a function of the colloidal volume fraction $\phi_{v}=4 \pi a^{3} n_{p} / 3$ by solving eq. (6) numerically and inserting $\phi(r)$ into eq. (7).

This procedure to obtain $f^{\text {ex }}$ from the nonlinear PB cell equation can be compared with linear PB cell theories. The "traditional" (Debye-Hückel or DLVO) way to linearize the PB 
equation is to replace $\sinh \phi \approx \phi$ in eq. (6), which is valid if $\phi \ll 1$. The resulting $f^{\text {ex }}$ is a simple analytic expression that follows directly from eq. (7) by expanding the term in square brackets to second order in $\phi$. Here, however, we propose an alternative, more general, linearization of eq. (6), based on the expansion $\sinh [\phi(\boldsymbol{r})]=\sinh [\bar{\phi}]+\cosh [\bar{\phi}](\phi(\boldsymbol{r})-\bar{\phi})+\ldots$, with $\bar{\phi}$ a constant to be determined. Insertion of this expansion into eq. (6), and neglecting $\mathcal{O}\left((\phi(\boldsymbol{r})-\bar{\phi})^{2}\right)$ terms yields

$$
\begin{aligned}
\nabla^{2} \tilde{\phi}(\boldsymbol{r}) & =\bar{\kappa}^{2}(\tanh [\bar{\phi}]+\tilde{\phi}(\boldsymbol{r})), & & \boldsymbol{r} \in G_{\text {cell }}, \\
\boldsymbol{n}_{1} \cdot \nabla \tilde{\phi} & =4 \pi \lambda_{\mathrm{B}} \sigma_{\mathrm{C}}, & & \boldsymbol{r} \in \partial G_{\boldsymbol{R}_{1}}, \\
\boldsymbol{n}_{1} \cdot \nabla \tilde{\phi} & =0, & & \boldsymbol{r} \in \partial G_{\text {cell }},
\end{aligned}
$$

with $\tilde{\phi}(\boldsymbol{r})=\phi(\boldsymbol{r})-\bar{\phi}$ and the modified screening parameter $\bar{\kappa}$ defined as

$$
\bar{\kappa}^{2}=\kappa^{2} \cosh \bar{\phi} \quad\left(\geq \kappa^{2}\right) .
$$

Clearly, eq. (8) reduces to the traditional linear PB equation if we set $\bar{\phi}=0$. Here we leave $\bar{\phi}$ unspecified for the moment and solve eq. (8) for arbitrary $\bar{\phi}$, resulting in

$$
\phi(\boldsymbol{r})=\bar{\phi}-\tanh [\bar{\phi}]-\frac{Z \lambda_{\mathrm{B}}}{r}\left[A(\bar{\kappa}) e^{-\bar{\kappa} r}+B(\bar{\kappa}) e^{+\bar{\kappa} r}\right],
$$

with $r=|\boldsymbol{r}|$ the radial coordinate. The coefficients $A(\bar{\kappa})=R^{-} / D$ and $B(\bar{\kappa})=-R^{+} / D$ are defined through the three relations $R^{ \pm}=\exp [\mp \bar{\kappa} R](1 \pm \bar{\kappa} R), a^{ \pm}=\exp [\mp \bar{\kappa} a](1 \pm \bar{\kappa} a)$ and $D=a^{+} R^{-}-R^{+} a^{-}$. If $\bar{\kappa} R \gg 1$, i.e., if the cell is much larger than the thickness of the double layer, we can ignore the existence of a cell of finite volume, and the coefficient $A(\bar{\kappa})$ reduces to $e^{\bar{\kappa} a} /(1+\bar{\kappa} a)$, while $B(\bar{\kappa})$ vanishes. In the following, we will refer to this approximation $\left(A(\bar{\kappa})=e^{\bar{\kappa} a} /(1+\bar{\kappa} a), B(\bar{\kappa})=0\right)$ as $\operatorname{Lin}_{A}$ scheme, while the symbol $\operatorname{Lin}_{A B}$ is used to refer to the full linear solution in eq. (10). Expanding $\phi$ about $\bar{\phi}$ in eq. (7) and inserting eq. (10) yields

$$
\begin{aligned}
\beta f_{\mathrm{lin}}^{\mathrm{ex}}= & \frac{Z^{2} \lambda_{\mathrm{B}}}{2 a}\left[A(\bar{\kappa}) e^{-\bar{\kappa} a}+B(\bar{\kappa}) e^{\bar{\kappa} a}-1\right]-Z(\bar{\phi}-\tanh \bar{\phi})+ \\
& +n_{s}^{r} \Delta_{r}[2-2 \cosh \bar{\phi}+\sinh \bar{\phi} \tanh \bar{\phi}]
\end{aligned}
$$

with $\Delta_{r}=4 \pi\left(R^{3}-a^{3}\right) / 3$ the reduced cell volume.

So far $\bar{\phi}$ was unspecified, but which linearization point $\bar{\phi}$ should we choose? As remarked already, we recover the traditional linearization by taking $\bar{\phi}=0$, but this does not seem to be a good choice in a concentrated colloidal suspension, where $\phi(\boldsymbol{r})$ hardly ever becomes zero. Here we suggest to linearize about the Donnan potential, which is defined as the average of $\phi(\boldsymbol{r})$ over the region outside the colloidal sphere,

$$
4 \pi \int_{a}^{R} \mathrm{~d} r r^{2}[\phi(\boldsymbol{r})-\bar{\phi}]=0 .
$$

Inserting eq. (10) into eq. (12) and using eq. (9) yields

$$
\sinh \bar{\phi}=\frac{\phi_{v}}{1-\phi_{v}} \frac{(-3 Z)}{8 \pi n_{s}^{r} a^{3}},
$$

as a definition equation of $\bar{\phi}$. This choice has a number of interesting features. i) Linearizing the electro-neutrality condition around $\bar{\phi}$ and imposing eq. (12), one is led again to eq. (13). 
Hence, eq. (13) ensures that the electro-neutrality condition is always satisfied. ii) In the limit of low colloidal density (or high salt concentration) we obtain $\bar{\phi} \rightarrow 0$, i.e. we recover the traditional DH linearization scheme, as one would expect. iii) Using eq. (12), one can calculate the total number of ions in the cell, $4 \pi \int_{a}^{R} \mathrm{~d} r r^{2}\left(n_{-}+n_{+}\right) \approx 2 n_{s}^{r} \Delta_{r} \cosh \bar{\phi} \equiv 2 N_{s}+Z$, with $N_{s}$ the number of co-ions in the cell. Combining this with eq. (9) yields

$$
\bar{\kappa}^{2}=\frac{4 \pi \lambda_{\mathrm{B}}\left(2 N_{s}+Z\right)}{\Delta_{r}},
$$

which implies that linearization around the Donnan potential leads to a screening constant that involves the total microion density in the system, including the counterions! This is a crucial difference with a linearization about $\bar{\phi}=0$, where the reservoir salt concentration governs the screening constant for any $n_{p}$. Note that the counterion contribution to the screening is often either ignored or put in by hand. Here we derived that ignoring it corresponds to a linearization of the PB equation about $\bar{\phi}=0$, whereas including it stems from a linearization about the self-consistently determined average potential. iv) Combining the expressions for $\cosh \bar{\phi}$ and $\sinh \bar{\phi}$, and using that $\cosh ^{2} \bar{\phi}-\sinh ^{2} \bar{\phi}=1$, leads to $N_{s}=-Z / 2+\left(n_{s}^{r 2} \Delta_{r}^{2}+Z^{2} / 4\right)^{1 / 2}$, the standard expression for the Donnan effect.

Let us now compare $f^{\text {ex }}$ that results from the traditional Debye-Hückel (DH) linearization $(\bar{\phi}=0)$ with that from linearization about the Donnan potential ( $\bar{\phi}$ from eq. $(13))$. In the DH scheme the excess free energy in eq. (11) reads $\beta f_{\mathrm{DH}}^{\mathrm{ex}}=Z^{2} \lambda_{\mathrm{B}}\left(A(\kappa) e^{-\kappa a}+B(\kappa) e^{\kappa a}-1\right) / 2 a$, which reduces to the DH self-energy $\beta f_{\mathrm{DH}}^{\text {self }}=-Z^{2} \lambda_{\mathrm{B}} \kappa /(2(1+\kappa a))$ of standard DH theory if the cell is ignored $\left(\operatorname{Lin}_{A}\right.$ scheme). Linearization about the Donnan potential yields

$$
\begin{aligned}
\beta f_{\text {Don, } A B}^{\text {ex }}= & \frac{Z^{2} \lambda_{\mathrm{B}}}{2 a}\left[A(\bar{\kappa}) e^{-\bar{\kappa} a}+B(\bar{\kappa}) e^{\bar{\kappa} a}-1\right]-\frac{2 \pi Z^{2} \lambda_{\mathrm{B}}}{\Delta_{r} \bar{\kappa}^{2}}+ \\
& +Z \ln \frac{N_{s}+Z}{n_{s}^{r} \Delta_{r}}-\left(2 N_{s}+Z-2 n_{s}^{r} \Delta_{r}\right),
\end{aligned}
$$

which reduces to

$$
\beta f_{\text {Don, } A}^{\text {ex }}=-\frac{Z^{2} \lambda_{\mathrm{B}} \bar{\kappa}}{2(1+\bar{\kappa} a)}-\frac{2 \pi Z^{2} \lambda_{\mathrm{B}}}{\Delta_{r} \bar{\kappa}^{2}}+Z \ln \frac{N_{s}+Z}{n_{s}^{r} \Delta_{r}}-\left(2 N_{s}+Z-2 n_{s}^{r} \Delta_{r}\right)
$$

in the $\operatorname{Lin}_{A}$ approximation, since then $A(\bar{\kappa})=e^{\bar{\kappa} a} /(1+\bar{\kappa} a)$ and $B(\bar{\kappa})=0$. The first term in eq. (16) has the same form as the DH self-energy, with the important difference that it depends on $\bar{\kappa}$ and hence on $n_{p}$. This term and the second one in eq. (16) are the long-known volume terms [11], which were shown to drive a spinodal instability and phase separation in refs. $[6,8]$. Here they follow easily from the Donnan linearization scheme in the cell geometry, which is the reason why we consider the $\operatorname{Lin}_{A}$ scheme here. The final two terms in eq. (16) are ideal-gas contributions stemming from the fact that the number density in the cell differs from that in the reservoir. In fig. 1a we compare, for $Z=100,1000$, and 3500 , the three expressions $\beta f_{\mathrm{DH}}^{\mathrm{ex}}, \beta f_{\mathrm{Don}, A B}^{\mathrm{ex}}$, and $\beta f_{\mathrm{Don}, A}^{\mathrm{ex}}$, based on linearized PB equations, with $\beta f^{\mathrm{ex}}$ that is based on the numerically solved nonlinear PB equation and eq. (7). We fixed the parameters $n_{s}^{r}=3.6 \mu \mathrm{M}, \lambda_{\mathrm{B}}=7.2 \AA$, and $a=133 \mathrm{~nm}$, for which a gas-liquid coexistence was predicted in ref. [6] for $Z=1217$. The first observation is that, for $Z=100$ and $Z=1000, \beta f_{\text {Don, } A B}^{\text {ex }}$ is an excellent approximation to $f^{\mathrm{ex}}$ of the nonlinear theory, except for a small deviation at low $\phi_{v}$ for $Z=1000$. This is to be contrasted with $\beta f_{\mathrm{Don}, A}^{\mathrm{ex}}$ and $\beta f_{\mathrm{DH}}^{\mathrm{ex}}$, which are much poorer, the latter especially for $Z=1000$ and 3500. So although the Donnan potential may not be the best linearization point, it is certainly much better than the DH linearization, expecially at high $\phi_{v}$ 

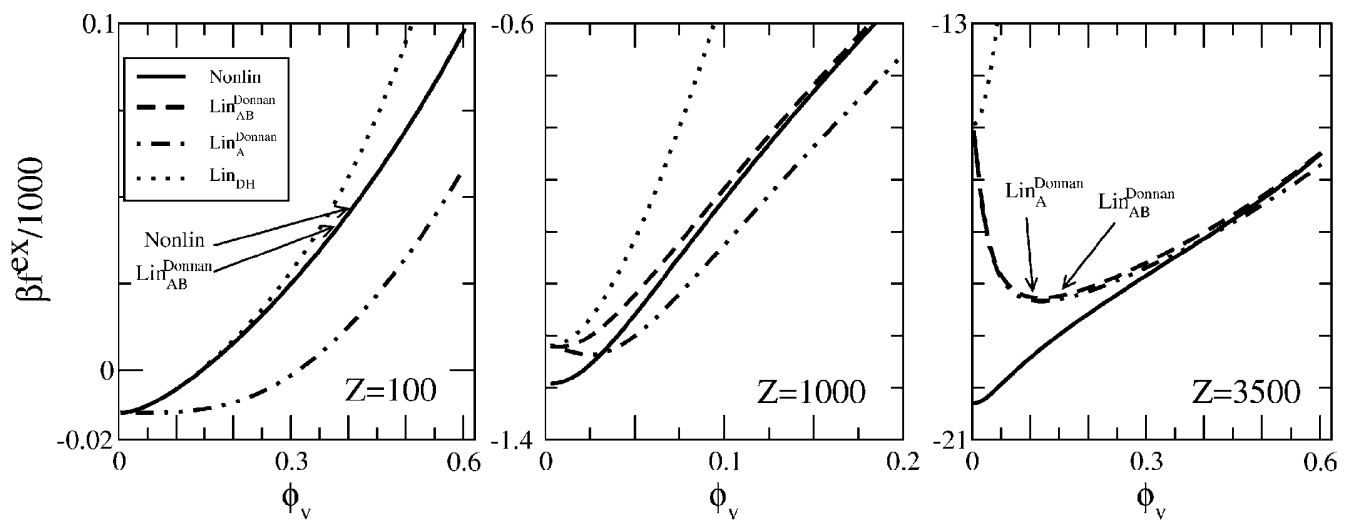

(a)
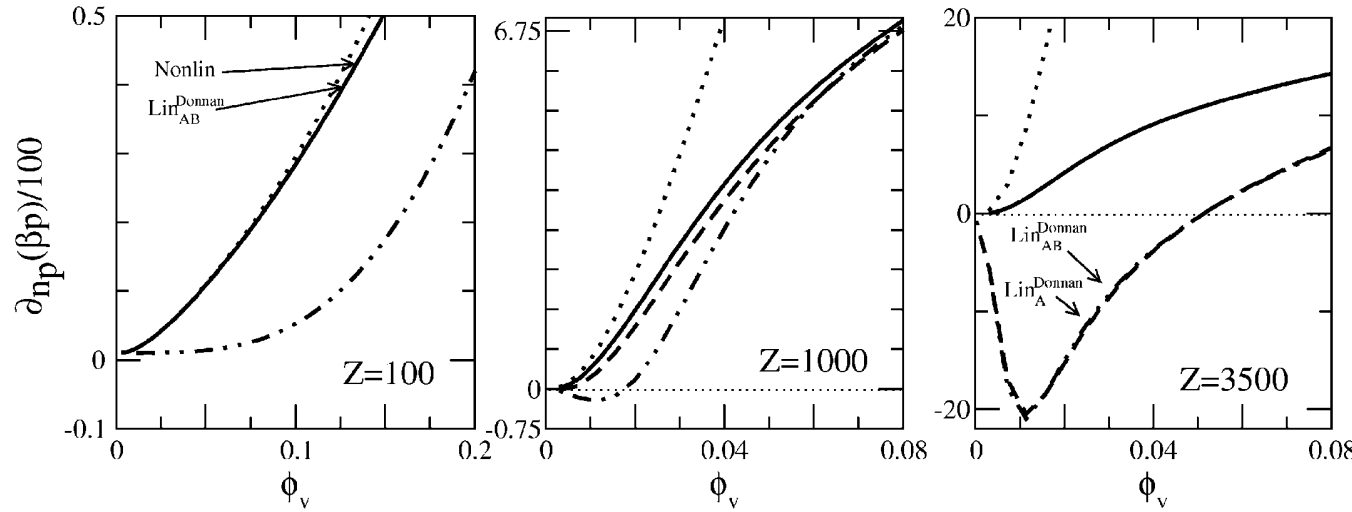

(b)

Fig. 1 - Excess free energy per particle (a) and compressibility (b) as a function of the colloidal packing fraction $\phi_{v}$ for colloidal charges $Z=100$ (left), 1000 (middle), 3500 (right). The full curves stem from the nonlinear Poisson-Boltzmann cell theory, and the dashed and dotted ones from several versions of linearized theories (see text).

and $Z$. Figure 1a actually suggests that there is a volume fraction $\phi_{v}^{c}$ beyond which the Donnan linearization becomes quantitatively accurate for a given $Z$; e.g., $\phi_{v}^{c} \simeq 0.15$ for $Z=1000$ and $\phi_{v}^{c}=0.5$ for $Z=3500$. For $\phi_{v}<\phi_{v}^{c}$ there is a considerable deviation between the Donnanlinearized and nonlinear theory. Note also that all three linear approximations coalesce to $\beta f_{\mathrm{DH}}^{\text {self }}$ for $\phi_{v} \rightarrow 0$, since $\bar{\phi} \rightarrow 0, \bar{\kappa} \rightarrow \kappa, A(\bar{\kappa}) \rightarrow A(\kappa)=e^{\kappa a} /(1+\kappa a)$ and $B(\bar{\kappa}) \rightarrow 0$ in this limit.

A typical feature of nonlinear PB theory is the charge accumulation at the colloidal surface ("ion condensation") $[12,13]$, which leads to a saturation behavior of the effective colloidal charge as a function of $Z$. This saturation behavior is not captured by linear theory, and therefore the DH self-energy $\beta f_{\mathrm{DH}}^{\text {self }}$ when seen as a function of $Z$, drastically fails for larger $Z$. We see that also in the three plots of fig. 1a, where the difference between linear and nonlinear theory at $\phi_{v}=0$ increases with increasing $Z$. Donnan linearization inherits this deficiency from the $\mathrm{DH}$ linearization, but becomes correct at large $\phi_{v}$. It is for this reason that $\beta f_{\mathrm{Don}, A B}^{\text {ex }}$ and $\beta f_{\mathrm{Don}, A}^{\mathrm{ex}}$ cross over to the incorrect $\mathrm{DH}$ result when $\phi_{v}$ is lowered from $\phi_{v}^{c}$ to 0 , with $\phi_{v}^{c}$ larger for larger $Z$. The negative slopes for higher $Z$ at low $\phi_{v}$ are due to the same deficiency.

In fig. $1 \mathrm{~b}$ we demonstrate that this low- $\phi_{v}$ deviation of $f_{\mathrm{Don}, A B}^{\mathrm{ex}}$ and $f_{\mathrm{Don}, A}^{\mathrm{ex}}$ from the nonlinear value $f^{\mathrm{ex}}$ gives rise to a spinodal instability and gas-liquid coexistence in the former and not in the latter case. The criterion for such an instability is that the compressibility $\left(\partial p / \partial n_{p}\right)_{\mu_{s}, T}<0$, with $p$ the osmotic pressure of the suspension given by $p=$ $n_{p}^{2}\left(\partial f / \partial n_{p}\right)_{\mu_{s}, T}$. In fig. 1b we plot $\partial_{n_{p}} \beta p=1+\partial_{n_{p}} n_{p}^{2} \partial_{n_{p}} \beta f^{\text {ex }}$ as a function of $\phi_{v}$, for the same 
parameters as in fig. 1a. The negative compressibilities that appear in fig. $1 \mathrm{~b}$ for $Z=1000$ and $Z=3500$ only appear in linear, but not in nonlinear theory. Although for $Z=1000$ only the $\operatorname{Lin}_{A}^{\text {Donnan }}$-scheme shows a negative compressibility, both Donnan-linearization schemes produce an instability for $Z=3500$. The two values of $\phi_{v}$ where the compressibility changes sign are the spinodal points, which for $Z=3500$ are located at $\phi_{v}=0.05$ and $\phi_{v}=10^{-4}$ (not to be seen on the scale of the plot). Repeating such a calculation for various $n_{s}^{r}$ yields a $\phi_{v}-n_{s}^{r}$ phase diagram with a closed-loop spinodal. Using the $\operatorname{Lin}_{A}^{\text {Donnan }}{ }_{\text {-scheme, we constructed a }}$ phase diagram for $Z=1217$ that showed a region of gas-liquid coexistence, consistent with that of ref. [6]. By contrast, within the nonlinear theory we did not find any indication of a phase instability; not a single combination of parameters $\left(Z, n_{s}^{r}, a\right)$ ever yielded a negative compressibility. On the basis of these cell calculations, one might therefore conclude that the failure of the linear theory at low $\phi_{v}$ is responsible for the prediction of a spurious gas-liquid instability in refs. $[6,8]$. We emphasize, however, that the present calculations are all based on the cell model, which constitutes an uncontrolled approximation that becomes less realistic and probably less reliable at low volume fractions. Note, however, that at $\phi_{v}=0$ the free energies (i.e. the self-energies) calculated in nonlinear theory are not dependent on the cell model approximation, because $R \rightarrow \infty$ as $\phi_{v} \rightarrow 0$. It should also be noted that the difference between the $\operatorname{Lin}_{A}$ and the $\operatorname{Lin}_{A B}$ scheme can be seen as a measure for the importance of the cell approximation. The fact that both schemes predict a phase instability and lead to very similar phase diagrams suggests that the cell approximation just makes a quantitative, but not a qualitative difference. We also emphasize that we have concentrated on monovalent ions. For divalent electrolyte ions electrostatic attraction between like-charged colloids is possible due to interionic correlations [14] (not captured in our mean-field approach) and has indeed been shown to produce a phase separation [15]. For a recent analysis similar to ours which is based on charge renormalization see also Diehl et al. [16].

$$
* * *
$$

It is a pleasure to thank M. Dijkstra, R. Evans, P. Warren, R. Klein, C. Fleck and J. P. HANSEN for stimulating discussions.

\section{REFERENCES}

[1] Larsen A. E. and Grier D. G., Nature, 385 (1997) 230.

[2] Ise N., Okubo T., Sugimura M., Ito K. and Nolte H. J., J. Chem. Phys., 78 (1983) 536.

[3] Trizac E., Phys. Rev. E, 62 (2000) R1465.

[4] Derjaguin B. and Landau L., Acta Phys. Chim. USSR, 14 (1941) 633; Verwey J. W. and Overbeek J. Тн. G., Theory of the Stability of Lyotropic Colloids (Elsevier, Amsterdam) 1948.

[5] Van Roij R. and Hansen J. P., Phys. Rev. Lett., 79 (1997) 3082.

[6] van Roij R., Dijkstra M. and Hansen J. P., Phys. Rev. E, 59 (1999) 2010.

[7] van Roij R. and Evans R., J. Phys. Condens. Matter, 11 (1999) 10047.

[8] Warren P. B., J. Chem. Phys., 112 (2000) 4683.

[9] HiLl T. L., An Introduction To Statistical Thermodynamics (Addison-Wesley Publishing Company) 1962.

[10] Belloni L., J. Phys. Condens. Matter, 12 (2000) R549.

[11] Beresford-Smith B., Chan D. Y. C. and Mitchell D. J., J. Colloid Interface Sci., 105 (1985) 215.

[12] Alexander S. et al., J. Chem. Phys., 80 (1984) 5776.

[13] Groot R. D., J. Chem. Phys., 95 (1991) 9191.

[14] Allahyarov E., Löwen H. and Trigger T., Phys. Rev. E, 57 (1998) 5818.

[15] Linse P. and Lobaskin V., J. Chem. Phys., 112 (2000) 3917.

[16] Diehl A., Barbosa M. C. and Levin Y., Europhys. Lett., 53 (2001) 86. 